\title{
Prior exposure to extreme pain alters neural response to pain in others
}

\author{
Moranne Eidelman-Rothman ${ }^{1}$ - Abraham Goldstein ${ }^{1,2}$ • Omri Weisman ${ }^{2}$. \\ Inna Schneiderman ${ }^{1}$ - Orna Zagoory-Sharon ${ }^{1} \cdot$ Jean Decety $^{3} \cdot$ Ruth Feldman $^{1,2}$
}

Published online: 31 March 2016

(C) Psychonomic Society, Inc. 2016

\begin{abstract}
In the extant literature examining the brain mechanisms implicated in pain perception, researchers have theorized that the overlapping responses to pain in the self and in others mark the human capacity for empathy. Here we investigated how prior exposure to extreme pain affects pain perception, by assessing the dynamics of pain processing in veterans who were previously exposed to severe injury. Fortythree participants (28 pain-exposed and 15 controls) underwent whole-head magnetoencephalography (MEG) while viewing photographs of limbs in painful and nonpainful (neutral) conditions. Among controls, an early (0-220 ms) "pain effect" in the posterior cingulate and sensorimotor cortices, and a later (760-900 ms) "pain effect" in the posterior cingulate cortex, superior temporal gyrus/insula, and fusiform gyrus were found, indicated by enhanced alpha suppression to the pain versus nonpain conditions. Importantly, pain-exposed participants exhibited an atypical pain response in the posterior cingulate cortex, indicated by a normative response to pain, but no pain-to-no-pain differentiation. This may suggest that individuals exposed to extreme pain may perceive neutral stimuli as potentially threatening. Our findings demonstrate alterations in pain perception following extreme pain
\end{abstract}

Electronic supplementary material The online version of this article (doi:10.3758/s13415-016-0422-7) contains supplementary material, which is available to authorized users.

Ruth Feldman

feldman.ruth@gmail.com

1 Gonda Multidisciplinary Brain Research Center, Bar-Ilan University, Ramat-Gan, Israel 52900

2 Department of Psychology, Bar-Ilan University, Ramat-Gan, Israel

3 Department of Psychology and Department of Psychiatry and Behavioral Neuroscience, University of Chicago, Chicago, IL, USA exposure, chart the sequence from automatic to evaluative pain processing, and emphasize the importance of considering past experiences in studying the neural response to others' states.

Keywords Pain perception · MEG $\cdot$ Alpha suppression · Posterior cingulate cortex

Understanding how the brain processes the experience of pain has played a key role in the field of social neuroscience. Research on pain perception in one's self and others has not only identified the brain networks activated when humans experience pain, but also showed overlapping activations to pain within the self and others (Y. Fan, Duncan, de Greck, \& Northoff, 2011; Lamm, Decety, \& Singer, 2011). Such overlap between the first- and third-person perspectives has provided the basis for much theorizing (Bastiaansen, Thioux, \& Keysers, 2009) and has highlighted the ability to represent others' states in one's own brain as the mechanism underpinning the human capacity for empathy (Decety \& Sommerville, 2003; Feldman, 2015).

The brain activation patterns associated with the first-hand experience of pain are well-defined, thus providing a reliable basis for comparing the brain response to pain in the self and others (Cheng, Yang, Lin, Lee, \& Decety, 2008). Functional magnetic resonance imaging (fMRI) studies have repeatedly shown that both the first-hand experience of pain and the observation of pain in others activate regions of the insula, cingulate cortex (Botvinick et al., 2005; Morrison, Lloyd, Pellegrino, \& Roberts, 2004), and sensorimotor cortex (Akitsuki \& Decety, 2009; Han et al., 2009), which are components of the pain-processing brain network (Peyron, Laurent, \& García-Larrea, 2000; Treede, Kenshalo, Gracely, \& Jones, 1999). The perception of pain in others also activates 
regions related to social information processing, such as the superior temporal sulcus/gyrus (STG; Botvinick et al., 2005; Singer et al., 2004) and the precuneus/posterior cingulate cortex (PCC), regions implicated in the ability to infer others' mental states (Saxe \& Wexler, 2005).

Whereas most studies have used fMRI, few have utilized electroencephalography (EEG) or magenetoencephalography (MEG) for assessing pain perception with higher temporal resolution. These studies have demonstrated that observing pain in others induces modulations in oscillatory brain activity in the alpha frequency range $(8-12 \mathrm{~Hz}$; Cheng et al., 2008; Mu, Fan, Mao, \& Han, 2008; Perry, Bentin, Bartal, Lamm, \& Decety, 2010; Whitmarsh, Nieuwenhuis, Barendregt, \& Jensen, 2011; Yang, Decety, Lee, Chen, \& Cheng, 2009). Oscillatory alpha activity is the predominant frequency in humans during rest and is suppressed in brain regions while processing relevant sensory information (Jensen \& Mazaheri, 2010; Klimesch, 2012). Thus, the suppression of alpha oscillations, associated with an asynchronous firing mode in the measured neural population, is thought to reflect enhanced activation in the corresponding cortices (Pfurtscheller, Stancák, \& Neuper, 1996). When recorded over sensorimotor cortices, alpha oscillations are termed $m u$ oscillations (Kuhlman, 1978). Two MEG studies (Cheng et al., 2008; Whitmarsh et al., 2011) showed mu suppression in responses to body parts in painful and nonpainful situations, which was significantly stronger in the pain condition. Comparable results have been found in EEG studies (Perry et al., 2010; Yang et al., 2009; but see Mu et al., 2008) using similar stimuli. In the Perry et al. study, the enhanced pain-related alpha suppression was not restricted to central regions, but was also found in frontal electrodes. Recently, reduced alpha activity was observed in regions of the PCC and in the fusiform gyrus (FG) to painful relative to nonpainful stimuli (Höfle, Pomper, Hauck, Engel, \& Senkowski, 2013). Thus, pain-related responses measured by alpha activity modulations have been observed in various brain regions and can provide valuable information regarding the time course of pain processing, which cannot be captured via fMRI.

Although research on pain perception in the self and others has generated valuable insights, several questions remain unexplored and require further research for theory-building. First, it is unclear whether prior exposure to extreme pain in one's self or in others alters the neural response to others' pain. Prior exposure to extreme pain may lead to desensitization, which may hamper motivation to detect pain in others (Franck $\&$ Bruce, 2009). Since fMRI studies typically compare brain responses to painful versus nonpainful but otherwise similar stimuli, it is unclear whether witnessing extreme pain would diminish or elevate responses to painful stimuli, or whether it would enhance or dampen the "pain effect"-defined as the difference in the responses to pain versus nonpain. Such findings suggest that research on the brain's responses to pain, and the theoretical implications thereof, must take into account an individual's history, and particularly salient pain-related experiences. In support, Teutsch, Herken, Bingel, Schoell, and May (2008) showed that continuous exposure to pain in the self led to substantial structural alterations in brain regions associated with pain processing, and a recent fMRI study in which painful stimuli preceded presentation of visual painrelated stimuli demonstrated that prior pain exposure decreased activity in the brain regions associated with observed pain (Preis, Schmidt-Samoa, Dechent, \& Kroener-Herwig, 2013). Second, most pain studies have used fMRI technology, which has poor temporal resolution. Thus, the sequential pattern of the brain's response to pain has received little attention, particularly the differentiation between areas that activate automatically and those involved in later, more evaluative processing.

In this study, we utilized high-temporal-resolution MEG to investigate the neural response to others' pain. MEG uniquely combines high temporal and spatial resolution, and thus assesses both temporal dynamics and the origins of the measured signal. To date, only a few studies have investigated the perception of others' pain via MEG. Therefore, our first goal was to extend existing research by investigating the spatio-temporal dynamics of the typical response to others' pain as measured in normative individuals. Next, we investigated veterans who had been exposed to serious injury in others in the course of combat to examine how previous exposure to pain-related traumatic experiences affects the typical neural response to others' pain. Since no study, to our knowledge, has used stimuli depicting others in physical pain to examine information processing in combat-exposed individuals, such an investigation may provide additional information on the neural responses associated with combat exposure. We utilized a set of well-validated stimuli showing hands and feet in painful and nonpainful situations (Jackson, Meltzoff, \& Decety, 2005) for the assessment of pain perception, and were specifically interested in measuring alpha activity modulations and defining their time course and origin in the brain.

The following hypotheses were formulated. First, in the nonexposed group the typical response to pain would be found expressed in enhanced alpha suppression to painful as compared to neutral stimuli in brain regions associated with pain perception and social information processing. Second, prior exposure to others' pain would alter the brain response to pain-related stimuli. Although stimuli depicting others in painful and nonpainful conditions have not been used in combat-exposed individuals, reduced discrimination in response to aversive versus neutral stimuli has recently been demonstrated in individuals with posttraumatic stress disorder (PTSD) due to combat exposure (MacNamara, Post, Kennedy, Rabinak, \& Phan, 2013). We therefore speculated that the group of pain-exposed individuals would show decreased differentiation in response to the pain versus nonpain conditions. 


\section{Materials and method}

\section{Participants}

We recruited a group of young (age $<35$ years), physically healthy male veterans in the Israel Defense Force. This included a group of 28 veterans (age range 23 to 31 years, mean $=27$ years, $S D=1.8$ ) who served in active combat units during the past 8 years (between 36 and 72 months) and witnessed the injury or death of another person (pain-exposed), and 16 control veterans (age range 24 to 34 years, mean $=28.93, S D=3.02$ ) who served during the same period in noncombat units (intelligence or technical support) and did not participate in active combat. All of the veterans completed the Post-Traumatic Stress Diagnostic Scale (PDS; Foa, Cashman, Jaycox, \& Perry, 1997) for the assessment of posttraumatic symptoms. Pain-exposed individuals showed moderate-to-severe PDS symptoms (mean $=16.13$, $S D=6.98)$, and the controls showed no posttraumatic symptoms $($ mean $=0)$. The participants were right-handed as measured by the Edinburgh Handedness Questionnaire, except for three pain-exposed participants who were ambidextrous $(N=1)$ or left-handed $(N=2)$. Exclusion criteria included serious physical injury, current or past neurological disorders, serious medical problems, and the regular use of medication. All participants provided written informed consent to participate in the study, which was approved by the local institutional review board.

\section{Procedure}

Each participant took part in two similar experimental sessions approximately a week apart $(M=7.5$ days, $S D=2)$. In each session, baseline blood and saliva samples were collected, followed by self-administration of either the neuropeptide oxytocin (OT) or placebo (PBO), as previously described (Eidelman-Rothman et al., 2015). Approximately 45 min after $\mathrm{OT} / \mathrm{PBO}$ administration, brain activity was measured using MEG in response to a well-validated set of colored photographs showing human right hands and feet in painful and nonpainful situations (Jackson et al., 2005). For each photograph depicting a painful situation, a corresponding one showed a neutral situation involving the same setting without any painful component (Fig. 1a). To avoid the influence of processes related to stimulus familiarity, learning, and memory, a pool of 80 photographs was divided into two nonidentical sets of 40 stimuli, including 20 matched pairs of painful and nonpainful stimuli. Each set was chosen to contain ten matched pairs of legs and ten matched pairs of hands in painful and nonpainful conditions, which were randomly assigned to the different stimulus sets. For each participant, a different set of stimuli was randomly assigned to each MEG session (the PBO or OT session), and the order of the stimulus sets presented was counterbalanced across participants. Each stimulus was displayed for $200 \mathrm{~ms}$, consistent with previous research (Y. Fan \& Han, 2008), followed by a fixation cross, during which the participants judged by pressing a button (using the right index finger) whether the photograph depicted a painful or a nonpainful situation. Following the response, the fixation cross remained on the screen for a duration varying randomly between 1,000 and 1,600 ms (Fig. 1b). The stimuli were presented using the E-Prime software (Psychology Software Tools, Inc.) in four experimental blocks (80 stimuli per block in a random order), with a short break between them, on a 17 -in. screen located $60 \mathrm{~cm}$ in front of the participant. The size of all stimuli was $15.87 \times 11.96 \mathrm{~cm}$. Two additional saliva samples were collected in each experimental session, one before and one after the MEG scanning. After the scanning, participants were asked to rate the pain intensity felt by the person in the photograph as well as their own unpleasantness, on the same set of stimuli presented during the MEG scan. The ratings were measured using a 6 -point scale $(1=$ no pain/not unpleasant, $6=$ very painful/very unpleasant $)$.

\section{Brain activity recording and analysis}

MEG recordings were conducted (sampling rate of $1017 \mathrm{~Hz}$, online 1 - to $400-\mathrm{Hz}$ band-pass filter) with a whole-head, 248channel magnetometer array (4-D Neuroimaging, Magnes $3600 \mathrm{WH}$ ) in a supine position, inside a magnetically shielded room. Reference coils located a short distance $(\sim 30 \mathrm{~cm})$ away from the 248 sensors and oriented in the $x, y$, and $z$ axes were used to record environmental noise. Head position was indicated by attaching five coils to the scalp and determining their positions relative to the sensor array. External noise (e.g., power-line or mechanical vibrations) and heartbeat artifacts were removed from the data using a predesigned algorithm, described previously (Tal \& Abeles, 2013). Signal preprocessing and analysis at the sensor level were carried out using MATLAB (The Mathworks, Natick, MA) and the FieldTrip toolbox (Oostenveld, Fries, Maris, \& Schoffelen, 2010). The data were segmented into 2,000-ms epochs, starting $800 \mathrm{~ms}$ before stimulus presentation and ending at 1,200 ms following stimulus presentation. Each data epoch was visually inspected for muscle artifacts and power jumps, which were excluded from further analysis. The segmented trials were then bandpass filtered in the $1-$ to $40-\mathrm{Hz}$ range with $10 \mathrm{~s}$ padding, and were baseline-corrected by subtracting the mean amplitude of the prestimulus period of $300 \mathrm{~ms}$ before each trial from all of the data points in the segment. Finally, spatial independent component analyses were applied in order to identify and remove eye movements, blinks, or other potential artifacts from the data. Two MEG sensors were excluded from the analyses due to malfunction. One participant from the control group and two from the pain-exposed group were excluded 
Fig. 1 The experimental paradigm. a Visual stimuli depicting nonpainful and painful situations. b The experimental trial design

\section{a}

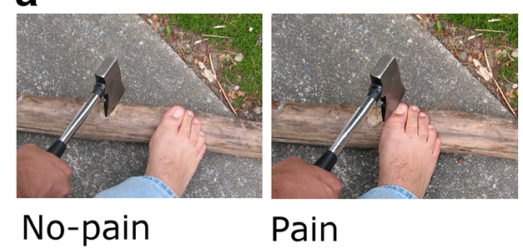

b

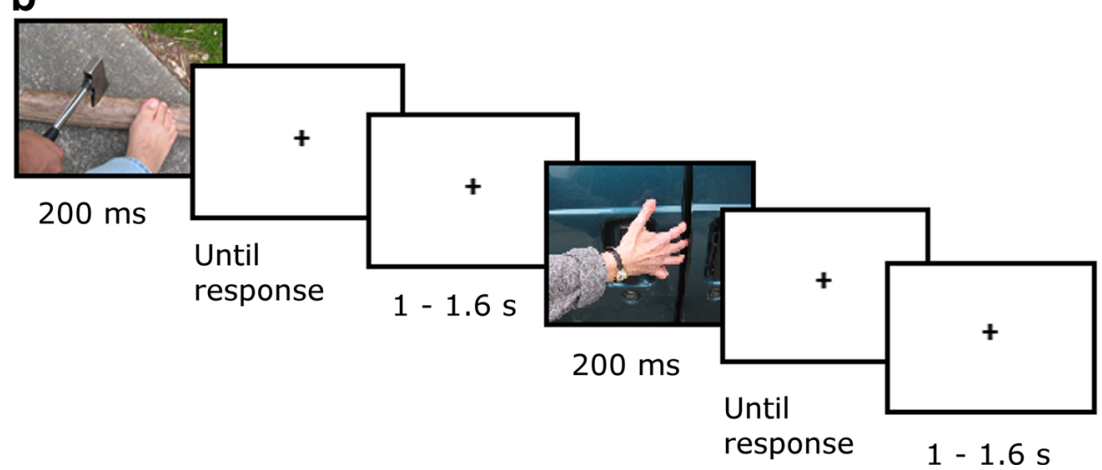

from all analyses due to excessive heartbeat MEG artifacts, which could not be removed from the data.

A time-frequency analysis was then performed at the sensor level to verify that the previously documented "pain effect" (i.e., increased response to pain vs. nonpain) was evident in our control-group data. In addition, this effect was used to define the specific time course of the "pain effect" for the subsequent source-level analysis (see below). Each data segment recorded during the PBO session was subjected to spectral analysis at the sensor level. A Hanning taper was applied to each epoch of the 246-sensor data to calculate the fast Fourier transform in the 1- to $40-\mathrm{Hz}$ frequency range, with short sliding time windows of $0.5 \mathrm{~s}$ in steps of $30 \mathrm{~ms}$.

For source estimation, a template MRI (Collin27) was modified to fit each participant's digitized head shape using SPM8 (Wellcome Department of Imaging Neuroscience University College London,www.fil.ion.ucl.ac.uk). A synthetic-aperture magnetometry (SAM) beamformer was used to estimate the neural sources of the measured MEG signal (Robinson \& Vrba, 1999). SAM acts as a spatial filter specific for each location in the head, to suppress the interference of the unwanted signals. SAM was applied to the data with a spatial resolution of $0.5 \mathrm{~cm}$ for each stimulus condition ("pain", "no-pain"), for time windows in which a sensor-level "pain effect" was observed (time windows of interest: TOIs). This analysis was performed on a standard alpha frequency range, $8-12 \mathrm{~Hz}$, since a wide frequency range is required for effective calculation of the data covariance, as part of the beamforming procedure. The spatial filter was applied for both the TOIs defined by the sensor-level analysis (0-220 ms and 760-900 ms following stimulus onset) and for the baseline data recorded over $300 \mathrm{~ms}$ before stimulus onset. The beamforming product of the baseline period was then subtracted from that of the TOIs to obtain a measure of alpha suppression for each trial. The baselinesubtracted trials in each TOI were averaged, and the data were presented as functional maps on each participant's modified template. These templates were then used to transform the beamforming functional images into a common Talairach space, and clusters with significant effects were then identified by means of voxel-level $t$ tests using the $3 \mathrm{dttest}$ module of the Analysis of Functional NeuroImages software (http://afni.nimh.nih.gov/afni). To control for the Type I error rate due to multiple comparisons over voxels, we applied a nonparametric permutation approach (Nichols \& Holmes, 2002) in which a $t$ test was repeated 1,000 times. In each permutation, the stimulus conditions ("pain" and "no-pain") were randomly assigned within subjects. For each permutation, the largest $5 \%$ of clusters of voxels with significant $t$ values $(p<.05)$ were recorded. A frequency distribution of these clusters was then used to determine the critical cluster size, which corresponded to the 50th maximal cluster size (5\%). Clusters with significant "pain effects" in controls at PBO in each TOI were defined as the regions of interest (ROIs) for testing group effects. For each participant, the voxel showing the minimal value, in each cluster and each condition, was selected for a statistical analysis that included repeated measures analysis of variance (ANOVA) followed by post-hoc $t$ tests. In the present study, we chose to focus on the PBO condition and to test group differences at baseline only, in light of the recent criticism on underpowered OT administration studies (Walum, Waldman, \& Young, 2016), and thus all following analyses are reported for the PBO condition. 


\section{Results}

\section{Task performance and subjective stimulus rating}

Mean response accuracy and reaction times were calculated for each participant in the pain judgment task (Supplementary Table 1). None of these measures showed differences related to group or stimulus condition ("pain"/“no-pain"). The selfreported pain intensity and unpleasantness ratings indicated that participants rated the painful stimuli significantly higher (mean pain intensity $=3.31, S D=0.81$; mean selfunpleasantness $=2.62, S D=1.26$ ) than the neutral stimuli (mean pain intensity $=0.13, S D=0.14$; mean self-unpleasantness $=0.27, S D=0.39$ ) on both measures $\left(p s=3.4 \times 10^{-24}\right.$ and $1.76 \times 10^{-15}$ for pain intensity and self-unpleasantness).

\section{Sensor-level analysis}

A sensor-level analysis for the control group revealed eventrelated alpha suppression along the trial period in both stimulus conditions ("pain," "no-pain"; Fig. 2, left and middle panels). Consistent with previous reports, painful stimuli induced stronger alpha suppression than did nonpainful stimuli ("pain" minus "no-pain"; Fig. 2, right panel). This "pain effect" was most notable in two distinct time windows: an early time window of 0-220 ms, and a later window of 760$900 \mathrm{~ms}$ following stimulus presentation. Since a Hanning window with a length of $0.5 \mathrm{~s}$ was used for the time-frequency analysis, the alpha suppression at each time point in the trial reflects a summary of the alpha suppression measured within a time window of $500 \mathrm{~ms}$ surrounding it. The late "pain effect" occurred earlier than the averaged reaction time calculated for the pain judgment task (Supplementary Table 1).

\section{Source-level analysis}

A source-level analysis conducted for the control group identified a significant early (0-220 ms) "pain effect" in two clusters of activity, which included the left PCC and the right sensorimotor cortex (paracentral lobule; Fig. 3, upper panel and Table 1) and a later (760-900 ms) "pain effect" in four clusters, which included the right PCC, bilateral FG/middle occipital gyrus (MOG), and the left STG/insula (Fig. 3, lower panel and Table 2). In the late time window, a significant cluster of activity was found in the right supramarginal gyrus $(x, y, z$ of peak MNI coordinates $=64-4527)$ in which no "pain effect" was detected. Alternatively, an opposite effect of enhanced alpha suppression in the neutral as compared to the painful condition was detected. Brain regions showing the "pain effect" in each time window were used as the ROIs for the analysis of group differences.

A repeated measures ANOVA with Pain Status (pain, nopain) as a within-subjects factor and Group as betweensubjects factor was applied for each region. In the early time window $(0-220 \mathrm{~ms})$, a main effect $[F(1,38)=5.779, p=.02]$ for pain status, as well as a Group $\times$ Pain Status interaction effect $[F(1,38)=4.139, p=.049]$, was found in the left PCC, indicating that the changes in alpha suppression patterns between the "pain" and "no-pain" conditions differed by groups (Fig. 4, left panel). Post-hoc $t$ tests showed that, whereas in the control group alpha suppression was stronger in the pain condition $[t(13)=3.81, p=.002]$, in the pain-exposed group no significant differences in activity were observed between the two stimulus conditions. In the right sensorimotor cortex, no main effect of pain exposure or significant Group $\times$ Pain interaction effect was found.

In the late time window (760-900 ms), main effects of pain exposure were found in all ROIs: the right PCC cluster $[F(1,38)=4.531, p=.04]$, the right $\mathrm{FG} / \mathrm{MOG}$
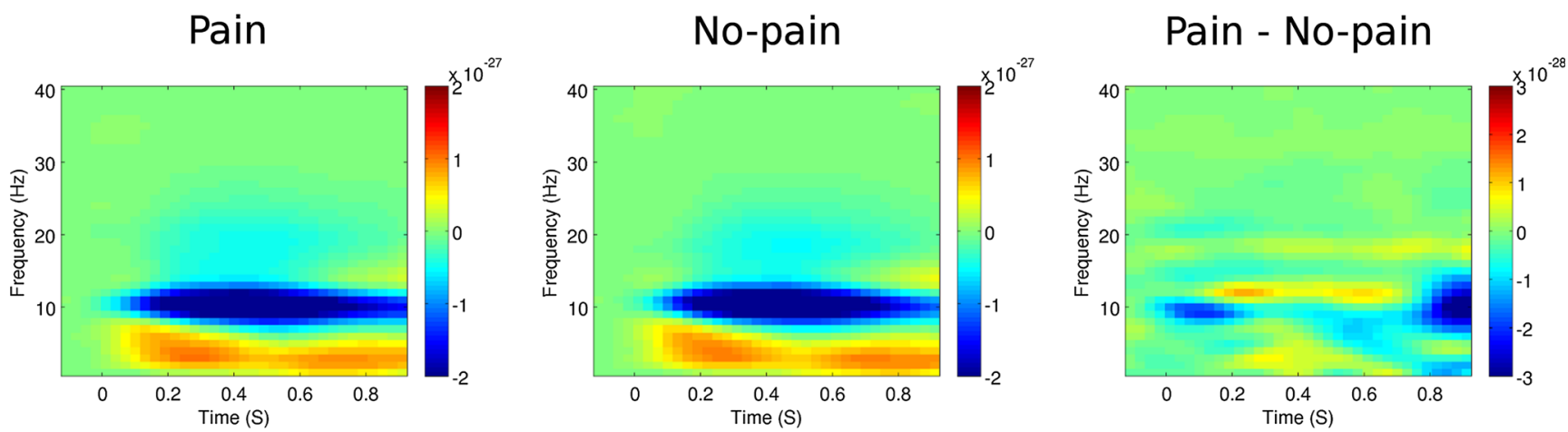

Fig. 2 Oscillatory brain responses to stimuli in the pain perception task: Time-frequency representations of the induced MEG responses elicited by the "pain" (left panel) and "no-pain" (middle panel) stimuli in the control group. Stimuli were presented at time 0. Alpha suppression (a decrease in alpha power, cooler shades) is seen in response to the stimuli in both conditions. A stronger suppression in response to "pain" relative to "no-pain" stimuli is seen in the alpha range, indicated by the negative result of subtracting the "no-pain" responses from the "pain" responses (right panel, cooler shades) 


\section{Early "pain effect" (0-220 ms)}

PCC

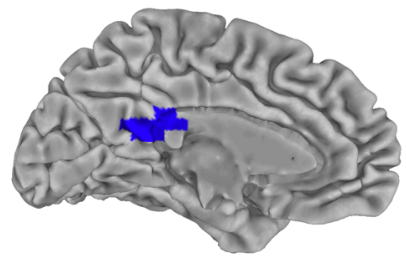

Sensorimotor Cortex

Late "pain effect" (760-900 ms)

PCC

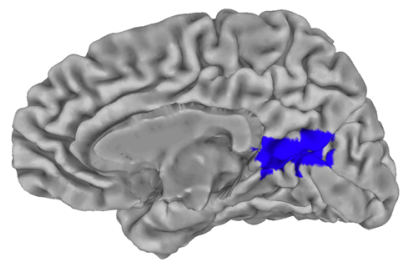

STG

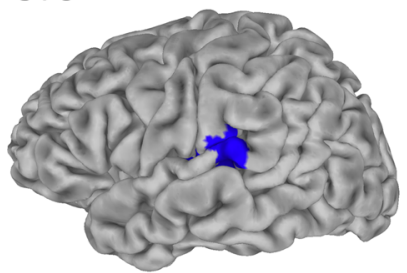

FG (R)

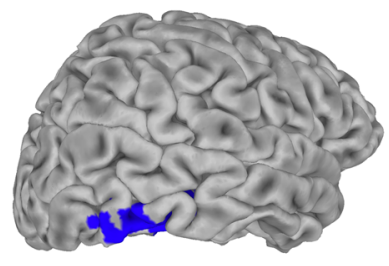

FG (L)

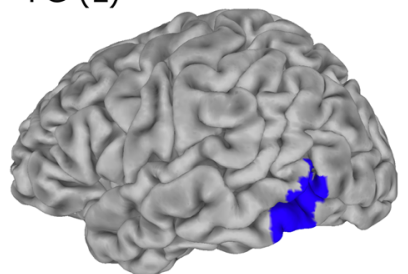

Fig. 3 Clusters showing a "pain effect" in the control group in the early (0-220 ms) and late (760-900 ms) time windows. Clusters in which alpha suppression was significantly stronger in the "pain" condition than in the "no-pain" condition (shown in color) were identified by a voxel-level $t$ test $(p<.05$, corrected) in the source estimation analysis. Peak $t$ values (the most significant voxels) were located in the left posterior cingulate cortex (PCC) and right paracentral lobule (sensorimotor cortex) in the early time window (upper panel), and in the right PCC, bilateral fusiform gyrus (FG), and left superior temporal gyrus (STG) in the late time window (lower panel)

Table 1 Clusters showing a significant "pain effect" in the early time window $(0-220 \mathrm{~ms})^{\mathrm{a}}$

\begin{tabular}{llllll}
\hline Cluster \# & Anatomical Region & \multicolumn{2}{c}{ MNI Coordinates } & \multirow{2}{*}{$t$ Value } \\
\cline { 3 - 4 } & & $x$ & $y$ & \multirow{2}{*}{$z$} & \\
\hline 1 & $\begin{array}{l}\text { L Posterior cingulate cortex } \\
\text { L Cingulate gyrus }\end{array}$ & -12 & -44 & 22 & 2.61 \\
& $\begin{array}{l}\text { L Middle temporal gyrus } \\
\text { R Paracentral lobule }\end{array}$ & 8 & -36 & 60 & 2.82 \\
\hline
\end{tabular}

${ }^{\text {a }} T$ values at peak coordinates (most significant voxels) are shown for clusters with a significant effect for pain (i.e., alpha suppression in the "pain" condition > alpha suppression in the "no-pain" condition) in the control group, as identified by a voxel-level $t$ test ( $p<.05$, corrected).

${ }^{\mathrm{b}}$ Montreal Neurological Institute (MNI) coordinates

Table 2 Clusters showing a significant "pain effect" in the late time window (760-900 ms)

\begin{tabular}{|c|c|c|c|c|c|}
\hline \multirow[t]{2}{*}{ Cluster \# } & \multirow[t]{2}{*}{ Anatomical Region } & \multicolumn{3}{|c|}{ MNI Coordinates ${ }^{b}$} & \multirow[t]{2}{*}{$t$ Value } \\
\hline & & $x$ & $y$ & $z$ & \\
\hline 1 & $\begin{array}{l}\text { R Posterior cingulate cortex } \\
\text { L Middle temporal gyrus } \\
\text { L Cuneus } \\
\text { L Posterior cingulate cortex } \\
\text { L Precuneus } \\
\text { L Superior occipital gyrus } \\
\text { R Precuneus }\end{array}$ & 8 & -49 & 5 & 3.37 \\
\hline 2 & $\begin{array}{l}\text { R Fusiform gyrus } \\
\text { R Middle occipital gyrus }\end{array}$ & 43 & -58 & -13 & 3 \\
\hline 3 & $\begin{array}{l}\text { L Superior temporal gyrus } \\
\text { L Insula } \\
\text { L Tranverse temporal gyrus }\end{array}$ & -63 & -34 & 17 & 3.64 \\
\hline 4 & $\begin{array}{l}\text { L Fusiform gyrus } \\
\text { L Middle occipital gyrus } \\
\text { Inferior temporal gyrus } \\
\text { Middle temporal gyrus }\end{array}$ & -53 & -69 & -2 & 3.25 \\
\hline
\end{tabular}

${ }^{a} T$ values at peak coordinates (most significant voxels) are shown for clusters with a significant effect for pain (i.e., alpha suppression in the "pain" condition > alpha suppression in the "no-pain" condition) in the control group, as identified by a voxel-level $t$ test $(p<.05$, corrected). ${ }^{\mathrm{b}}$ Montreal Neurological Institute (MNI) coordinates

$[F(1,38)=5.585, p=.023]$ and left $\mathrm{FG}[F(1,38)=4.096$, $p=.05]$ clusters, and the $\mathrm{STG} /$ insula cluster $[F(1,38)=5.750$, $p=.022]$, with greater alpha suppression overall in the "pain" condition. A Group $\times$ Pain interaction effect was observed only in the PCC cluster $[F(1,38)=11.75, p=.001]$. In this region, a significant difference in activity between the painful and neutral conditions once again was found only in the control group, such that alpha suppression was stronger in the "pain" condition than in the "no-pain" condition $[t(13)=3.4, p=.005]$. In addition, a follow-up $t$ test revealed between-group differences in which alpha suppression in the "no-pain" condition was significantly lower in the control group than in the pain-exposed group $[t(1,37)=2.97, p=.005]$ in the PCC (Fig. 4, right panel).

Overall, our findings indicate that differences in brain activation to "pain" and "no pain" in the PCC emerged only among controls. This suggests that prior exposure to extreme pain alters the typical brain response to pain by blurring the distinction between painful and otherwise identical but nonpainful stimuli, and that this blurring of the "pain effect" stems from increased responses to "no pain" rather than from attenuated response to pain. 
PCC early response (0-220 ms)

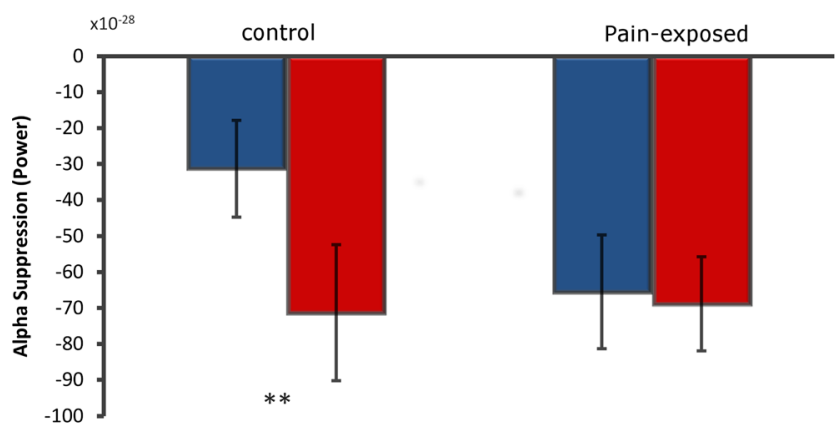

PCC late response (760-900 ms)

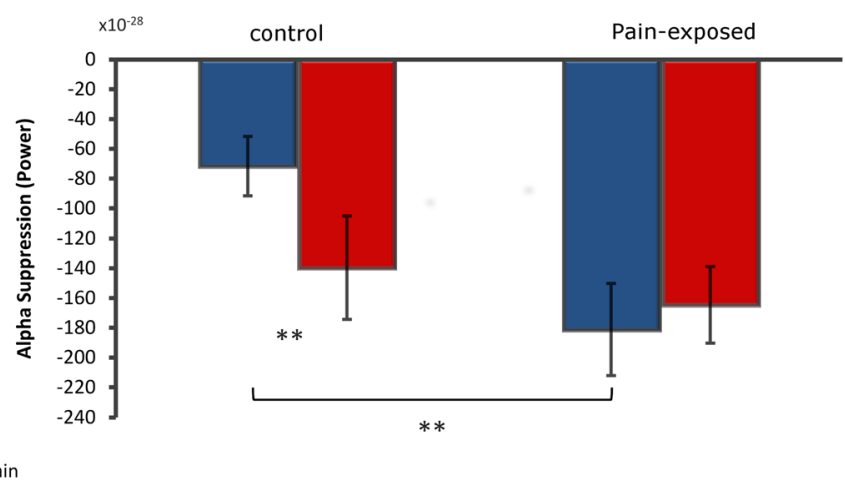

Fig. 4 Group and stimulus condition differences in alpha suppression in the early $(0-220 \mathrm{~ms})$ and late $(760-900 \mathrm{~ms})$ time windows. Mean alpha suppression in the early (left panel) and

\section{Discussion}

In the present study, we sought to extend existing research on the perceptual processing of pain by assessing the temporal patterns of the neural response to pain and by utilizing a special cohort of individuals who had previously been exposed to severe pain in others. By investigating individuals who had witnessed injury and pain in others, we were able to examine how such experiences may affect a basic, evolutionary-based response that is crucial for survival and supports the capacity to live within social groups.

Consistent with previous studies of pain perception (Cheng et al., 2008; Whitmarsh et al., 2011) and the attention-drawing nature of survival-related stimuli (Bradley, 2009), we found a "pain effect" in the control group marked by significantly greater alpha suppression in the painful than in the neutral condition in the PCC. This pattern, which may reflect enhanced evaluation of the more important stimulus (i.e., pain), was altered in the pain-exposed group, suggesting that prior exposure to severe pain in others is associated with alterations to the neural response to pain-related stimuli in specific brain areas. By using high temporal- and spatial-resolution MEG recordings, we were able to determine the time course of the "pain effect" found in the control group and to localize its sources in the brain. This included an early response, within the first $220 \mathrm{~ms}$ following stimulus onset, which was localized to the PCC and the sensorimotor cortex, and a later response, 760-900 ms following stimulus onset, located at the PCC, STG/insula, and occipital regions, including bilateral FG. The latter response preceded the participants' behavioral reaction in the pain judgment task, and might therefore be associated with stimulus evaluation. These findings are in line with those from a previous study that had used a similar experimental design and a pain judgment task to demonstrate an early and a late response to observed pain, and showed that the latter was modulated by attention to pain during task performance (Y. Fan \& Han, 2008). Such findings are in late (right panel) responses to "pain" and "no-pain" stimuli is shown for each experimental group in the posterior cingulate cortex (PCC)

agreement with perspectives suggesting two distinct components in the processing of others' state, including an early perceptual-attentional response and a later evaluative response (Cheng, Chen, \& Decety, 2014; Cheng, Hung, \& Decety, 2012). The findings are also compatible with previous E/MEG studies showing that early responses to others' pain can be observed within $200 \mathrm{~ms}$ following stimulus onset (Cheng et al., 2008; Y. Fan \& Han, 2008). In addition, our findings are in line with those from previous studies on the perception of others' pain that have shown activation in regions of the cingulate (Höfle et al., 2013) and sensorimotor (Cheng et al., 2008; Whitmarsh et al., 2011) cortices, as well as in posterior brain regions, including the FG (Höfle et al., 2013; Singer et al., 2004). Finally, similar to previous reports, we found a "pain effect" also in the STG (Botvinick et al., 2005; Singer et al., 2004), a region associated with salience (Hayes, Hayes, \& Mikedis, 2012; White, Joseph, Francis, \& Liddle, 2010) and social information processing (Bernhardt \& Singer, 2012), which was extended to the posterior insula, which is known to be associated with the sensory experience of pain (Peyron et al., 2000; Sabatini et al., 2009).

The pain-exposed group failed to show a "pain effect" in the PCC, where the difference in response to painful versus neutral stimuli significantly differentiated between groups. Although they demonstrated normative alpha suppression in response to pain, the pain-exposed group responded with similar levels of brain activation to the neutral stimuli in the later time window. The PCC has been previously linked with selfexperienced pain perception. Although some have reported pain-related deactivation of this region (Chae et al., 2013; Loggia et al., 2012), others have demonstrated pain-induced activity in the PCC (Bromm, 2001; Freund et al., 2010; Nielsen, Balslev, \& Hansen, 2005) which has been attributed by some to the emotional-aversive aspect of the painful experiences (Bromm, 2001). Recently, reduced alpha activity was found in the PCC in response to observed painful as compared to nonpainful stimuli (Höfle et al., 2013). Thus, the activation 
found in this region is consistent with the concept of shared neural representations for the observation of pain in others and its first-hand experience (Y. Fan et al., 2011; Lamm et al., 2011). The finding that pain-exposed individuals responded similarly to painful and neutral stimuli in the PCC may suggest that they perceived potential pain in the neutral condition, as well. It has been argued that the activation of brain regions attributed to pain processing is more broadly associated with the processing of sensory-salient information (Legrain, Iannetti, Plaghki, \& Mouraux, 2011). It has also been suggested that both painful and nonpainful aversive stimuli induce activation in a core aversion-related network (Hayes \& Northoff, 2012), which includes regions of the PCC and highlights its role in the basic processing of aversive stimuli. In addition, PCC activation has been associated with potential threat processing (Fiddick, 2011). The enhanced posterior cingulate responses measured in the pain-exposed group in the neutral condition may therefore be related to increased salience and threat processing. Since the salience of stimuli is thought to be determined, at least partly, by context and past experiences (Legrain et al., 2011), it is possible that the participants' previous pain-related experiences led to enhanced salience of the neutral stimuli and to increased attention to their potential threat. Thus, the enhanced responses to neutral stimuli may reflect overprocessing, which may be related to the survival importance of both pain and potential pain to combat-trained veterans. For such individuals, painful and neutral pictures may both be perceived as potentially threatening, especially within an experimental context in which painful and nonpainful stimuli appear interchangeably. It should be noted that the groups did not differ in task accuracy, and therefore the possible enhanced evaluation of the neutral stimuli in the pain-exposed group appears to be independent of response selection and execution.

Our findings demonstrate that prior exposure to severe pain and injury in others is associated with alterations in the neural response to others' pain in a brain region associated with pain perception and with aversive stimulus processing. These findings underscore the need to consider personal experiences when studying the neural underpinnings of pain perception. However, a number of limitations should be considered when interpreting the results. First, the pain-exposed participants showed posttraumatic symptoms, which may also be related to the observed alterations in the brain response to pain. Although inconsistency exists in the literature, alterations in the processing of aversive stimuli such as negative facial expressions have been demonstrated in combat-exposed individuals with (Catani, Adenauer, Keil, Aichinger, \& Neuner, 2009; MacNamara et al., 2013) and without (Catani et al., 2009) PTSD, including diminished differentiation in response to aversive and neutral stimuli (MacNamara et al., 2013). To distinguish activity patterns that are specific to pain processing from those more generally associated with the processing of aversive content, future studies of pain processing in combatexposed individuals will be required, which should also include negative stimuli that are not related to pain, such as threatening facial expressions, scenes, or events. In addition, a possible effect of preexisting differences between military personnel who have served in combat units and those who have not should also be considered. Second, to minimize inconvenience, we did not include pain threshold measurements. However, the participants' sensitivity to experienced pain may have had an effect on the processing of observed pain, similar to reports in other populations (Y.-T. Fan, Chen, Chen, Decety, \& Cheng, 2014). Next, as part of our experimental session design, a blood sample was collected from the participants shortly after arrival, which involves a mildly painful procedure. Although brain activity recordings were conducted approximately $45 \mathrm{~min}$ after the blood was drawn, the possibility that such a procedure impacted later pain processing and that this effect differed in the pain-exposed and control groups cannot be ruled out. Another methodological consideration that should be taken into account is related to our data analysis approach, in which the ROIs for the examination of pain processing in the pain-exposed group were defined on the basis of the results identified in the control group. This approach was chosen to demonstrate atypical pain processing relative to the normative response to pain measured by the MEG methodology. However, it is possible that our approach may have inflated error rates in the subsequent analyses conducted in the exposed group, and therefore replication of our findings in other trauma-exposed samples will be important. Finally, we did not detect pain-related activations in additional regions typically associated with pain perception, such as the anterior insula and ACC. This may be related to differences between the MEG and $\mathrm{AMRI}$ neuroimaging approaches. Using MEG, we were able to record earlier responses than those detected with fMRI, and future studies comparing the two methods are needed.

Although pain-related differences in brain activity were found between the groups, they were not accompanied by behavioral differences in the ratings of others' pain and selfunpleasantness. It is possible that other behavioral measures, such as measures that involve the estimation of potential threat and salience processing, could have revealed effects at the behavioral level and would have provided further insights, and such topics should be considered in future research. In addition, functional connectivity analysis may enhance our understanding of the spatio-temporal dynamics among the brain regions identified here. Much further research that includes in-depth assessments of personal history along with the collection of high spatial- and temporal-resolution data will be necessary for a better understanding of the neural mechanisms underpinning pain perception in the context of previous exposure to intense pain in the self and others. 
Author note This research was supported by a NARSAD independent investigator award, by the Simms-Mann Foundation, and by the I-CORE Program of the Planning and Budgeting Committee and the Israel Science Foundation (Grant No. 51/11). The authors have no conflict of interest to disclose.

\section{References}

Akitsuki, Y., \& Decety, J. (2009). Social context and perceived agency affects empathy for pain: An event-related fMRI investigation. NeuroImage, 47, 722-734.

Bastiaansen, J. A. C. J., Thioux, M., \& Keysers, C. (2009). Evidence for mirror systems in emotions. Philosophical Transactions of the Royal Society B, 364, 2391-2404. doi:10.1098/rstb.2009.0058

Bernhardt, B. C., \& Singer, T. (2012). The neural basis of empathy. Annual Review of Neuroscience, 35, 1-23. doi:10.1146/annurevneuro-062111-150536

Botvinick, M., Jha, A. P., Bylsma, L. M., Fabian, S. A., Solomon, P. E., \& Prkachin, K. M. (2005). Viewing facial expressions of pain engages cortical areas involved in the direct experience of pain. NeuroImage, 25, 312-319. doi:10.1016/j.neuroimage.2004.11.043

Bradley, M. M. (2009). Natural selective attention: Orienting and emotion. Psychophysiology, 46, 1-11. doi:10.1111/j.1469-8986.2008. 00702.x

Bromm, B. (2001). Brain images of pain. Physiology, 16, 244-249.

Catani, C., Adenauer, H., Keil, J., Aichinger, H., \& Neuner, F. (2009). Pattern of cortical activation during processing of aversive stimuli in traumatized survivors of war and torture. European Archives of Psychiatry and Clinical Neuroscience, 259, 340-351.

Chae, Y., Chang, D.-S., Lee, S. H., Jung, W. M., Lee, I. S., Jackson, S., \& Wallraven, C. (2013). Inserting needles into the body: A metaanalysis of brain activity associated with acupuncture needle stimulation. Journal of Pain, 14, 215-222.

Cheng, Y., Chen, C., \& Decety, J. (2014). An EEG/ERP investigation of the development of empathy in early and middle childhood. Developmental Cognitive Neuroscience, 10, 160-169.

Cheng, Y., Hung, A.-Y., \& Decety, J. (2012). Dissociation between affective sharing and emotion understanding in juvenile psychopaths. Development and Psychopathology, 24, 623-636.

Cheng, Y., Yang, C.-Y., Lin, C.-P., Lee, P.-L., \& Decety, J. (2008). The perception of pain in others suppresses somatosensory oscillations: A magnetoencephalography study. NeuroImage, 40, 1833-1840. doi:10.1016/j.neuroimage.2008.01.064

Decety, J., \& Sommerville, J. A. (2003). Shared representations between self and other: A social cognitive neuroscience view. Trends in Cognitive Sciences, 7, 527-533.

Eidelman-Rothman, M., Goldstein, A., Levy, J., Weisman, O., Schneiderman, I., Mankuta, D., \& Feldman, R. (2015). Oxytocin affects spontaneous neural oscillations in trauma-exposed war veterans. Frontiers in Behavioral Neuroscience, 9, 165. doi:10.3389/ fnbeh.2015.00165

Fan, Y.-T., Chen, C., Chen, S.-C., Decety, J., \& Cheng, Y. (2014). Empathic arousal and social understanding in individuals with autism: Evidence from fMRI and ERP measurements. Social Cognitive and Affective Neuroscience, 9, 1203-1213. doi:10.1093/ scan/nst101

Fan, Y., Duncan, N. W., de Greck, M., \& Northoff, G. (2011). Is there a core neural network in empathy? An fMRI based quantitative metaanalysis. Neuroscience \& Biobehavioral Reviews, 35, 903-911. doi: 10.1016/j.neubiorev.2010.10.009

Fan, Y., \& Han, S. (2008). Temporal dynamic of neural mechanisms involved in empathy for pain: An event-related brain potential study. Neuropsychologia, 46, 160-173.
Feldman, R. (2015). The adaptive human parental brain: Implications for children's social development. Trends in Neurosciences, 38 387-399.

Fiddick, L. (2011). There is more than the amygdala: Potential threat assessment in the cingulate cortex. Neuroscience \& Biobehavioral Reviews, 35, 1007-1018.

Foa, E. B., Cashman, L., Jaycox, L., \& Perry, K. (1997). The validation of a self-report measure of posttraumatic stress disorder: The Posttraumatic Diagnostic Scale. Psychological Assessment, 9, 445.

Franck, L. S., \& Bruce, E. (2009). Putting pain assessment into practice: Why is it so painful? Pain Research \& Management, 14, 13-20.

Freund, W., Wunderlich, A. P., Stuber, G., Mayer, F., Steffen, P., Mentzel, M., \& Schmitz, B. (2010). Different activation of opercular and posterior cingulate cortex (PCC) in patients with Complex Regional Pain Syndrome (CRPS I) compared with healthy controls during perception of electrically induced pain: A functional MRI study. Clinical Journal of Pain, 26, 339-347.

Han, S., Fan, Y., Xu, X., Qin, J., Wu, B., Wang, X., \& Mao, L. (2009). Empathic neural responses to others' pain are modulated by emotional contexts. Human Brain Mapping, 30, 3227-3237.

Hayes, D. J., \& Northoff, G. (2012). Common brain activations for painful and non-painful aversive stimuli. BMC Neuroscience, 13, 60.

Hayes, J. P., Hayes, S. M., \& Mikedis, A. M. (2012). Quantitative metaanalysis of neural activity in posttraumatic stress disorder. Biology of Mood and Anxiety Disorders, 2, 9. doi:10.1186/2045-5380-2-9

Höfle, M., Pomper, U., Hauck, M., Engel, A. K., \& Senkowski, D. (2013). Spectral signatures of viewing a needle approaching one's body when anticipating pain. European Journal of Neuroscience, 38, 3089-3098.

Jackson, P. L., Meltzoff, A. N., \& Decety, J. (2005). How do we perceive the pain of others? A window into the neural processes involved in empathy. NeuroImage, 24, 771-779.

Jensen, O., \& Mazaheri, A. (2010). Shaping functional architecture by oscillatory alpha activity: Gating by inhibition. Frontiers in Human Neuroscience, 4, 186. doi:10.3389/fnhum.2010.00186

Klimesch, W. (2012). Alpha-band oscillations, attention, and controlled access to stored information. Trends in Cognitive Sciences, 16, 606-617.

Kuhlman, W. N. (1978). Functional topography of the human mu rhythm. Electroencephalography and Clinical Neurophysiology, 44, 83-93.

Lamm, C., Decety, J., \& Singer, T. (2011). Meta-analytic evidence for common and distinct neural networks associated with directly experienced pain and empathy for pain. NeuroImage, 54, 2492-2502. doi:10.1016/j.neuroimage.2010.10.014

Legrain, V., Iannetti, G. D., Plaghki, L., \& Mouraux, A. (2011). The pain matrix reloaded: A salience detection system for the body. Progress in Neurobiology, 93, 111-124.

Loggia, M. L., Edwards, R. R., Kim, J., Vangel, M. G., Wasan, A. D., Gollub, R. L., \& Napadow, V. (2012). Disentangling linear and nonlinear brain responses to evoked deep tissue pain. Pain, 153, 2140-2151.

MacNamara, A., Post, D., Kennedy, A. E., Rabinak, C. A., \& Phan, K. L. (2013). Electrocortical processing of social signals of threat in combat-related post-traumatic stress disorder. Biological Psychology, 94, 441-449.

Morrison, I., Lloyd, D., di Pellegrino, G., \& Roberts, N. (2004). Vicarious responses to pain in anterior cingulate cortex: Is empathy a multisensory issue? Cognitive, Affective, \& Behavioral Neuroscience, 4, 270-278. doi:10.3758/CABN.4.2.270

Mu, Y., Fan, Y., Mao, L., \& Han, S. (2008). Event-related theta and alpha oscillations mediate empathy for pain. Brain Research, 1234, 128-136.

Nichols, T. E., \& Holmes, A. P. (2002). Nonparametric permutation tests for functional neuroimaging: A primer with examples. Human Brain Mapping, 15, 1-25. 
Nielsen, F. A., Balslev, D., \& Hansen, L. K. (2005). Mining the posterior cingulate: Segregation between memory and pain components. NeuroImage, 27, 520-532.

Oostenveld, R., Fries, P., Maris, E., \& Schoffelen, J. M. (2010). FieldTrip: Open source software for advanced analysis of MEG, EEG, and invasive electrophysiological data. Computational Intelligence and Neuroscience, 2011, 156869.

Perry, A., Bentin, S., Bartal, I. B.-A., Lamm, C., \& Decety, J. (2010). "Feeling" the pain of those who are different from us: Modulation of EEG in the mu/alpha range. Cognitive, Affective, \& Behavioral Neuroscience, 10, 493-504. doi:10.3758/CABN.10.3.493

Peyron, R., Laurent, B., \& García-Larrea, L. (2000). Functional imaging of brain responses to pain: A review and meta-analysis (2000). Clinical Neurophysiology, 30, 263-288.

Pfurtscheller, G., Stancák, A., Jr., \& Neuper, C. (1996). Event-related synchronization (ERS) in the alpha band-An electrophysiological correlate of cortical idling: A review. International Journal of Psychophysiology, 24, 39-46.

Preis, M. A., Schmidt-Samoa, C., Dechent, P., \& Kroener-Herwig, B. (2013). The effects of prior pain experience on neural correlates of empathy for pain: An fMRI study. Pain, 154, 411-418.

Robinson, S. E., \& Vrba, J. (1999). Functional neuroimaging by synthetic aperture magnetometry (SAM). In T. Yoshimoto, M. Kotani, S. Kuriki, H. Karibe, \& N. Nakasato (Eds.), Recent advances in biomagnetism: Proceedings of the 11th International Conference on Biomagnetism, Sendai, August 28-September 2, 1998 (pp. 302-305). Sendai: Tohoku University Press.

Sabatini, E., Penna, S. D., Franciotti, R., Ferretti, A., Zoccolotti, P., Rossini, P. M., \& Gainotti, G. (2009). Brain structures activated by overt and covert emotional visual stimuli. Brain Research Bulletin, 79, 258-264.
Saxe, R., \& Wexler, A. (2005). Making sense of another mind: The role of the right temporo-parietal junction. Neuropsychologia, 43, 1391-1399.

Singer, T., Seymour, B., O’Doherty, J., Kaube, H., Dolan, R. J., \& Frith, C. D. (2004). Empathy for pain involves the affective but not sensory components of pain. Science, 303, 1157-1161. doi:10.1126/ science. 1093535

Tal, I., \& Abeles, M. (2013). Cleaning MEG artifacts using external cues. Journal of Neuroscience Methods, 217, 31-38.

Teutsch, S., Herken, W., Bingel, U., Schoell, E., \& May, A. (2008). Changes in brain gray matter due to repetitive painful stimulation. NeuroImage, 42, 845-849.

Treede, R. D., Kenshalo, D. R., Gracely, R. H., \& Jones, A. K. P. (1999). The cortical representation of pain. Pain, 79, 105-111.

Walum, H., Waldman, I. D., \& Young, L. J. (2016). Statistical and methodological considerations for the interpretation of intranasal oxytocin studies. Biological Psychiatry, 79, 251-257.

White, T. P., Joseph, V., Francis, S. T., \& Liddle, P. F. (2010). Aberrant salience network (bilateral insula and anterior cingulate cortex) connectivity during information processing in schizophrenia. Schizophrenia Research, 123, 105-115.

Whitmarsh, S., Nieuwenhuis, I. L., Barendregt, H. P., \& Jensen, O. (2011). Sensorimotor alpha activity is modulated in response to the observation of pain in others. Frontiers in Human Neuroscience, 5, 91. doi:10.3389/fnhum.2011.00091

Yang, C. Y., Decety, J., Lee, S., Chen, C., \& Cheng, Y. (2009). Gender differences in the mu rhythm during empathy for pain: An electroencephalographic study. Brain Research, 1251, 176-184. doi:10.1016/j.brainres.2008.11.062 